\title{
LA UNIVERSIDAD POPULAR DE “LA VIGIL”, ROSARIO (1964-1977)
}

\author{
Natalia García (Universidad Nacional de Rosario - \\ Consejo Nacional de Investigaciones Científicas y Técnicas)* \\ nataliagr5@gmail.com
}

Recibido: 1\%/08/2012 Aceptado: 18/11/2012

\begin{abstract}
Resumen
El presente artículo tiene por objeto describir y analizar la experiencia de la Universidad Popular de la Biblioteca "Constancio C. Vigil" instituida en 1964 y clausurada en 1977 por la última dictadura cívico-militar argentina (1976-1983). En rigor de una investigación concluida sobre la historia de la organización en su conjunto, se considera significativo presentar los principales datos cuantitativos y cualitativos que abonan a una comprensión de los proyectos pedagógicos de "la Vigil" en su trama educativa no formal. Al respecto, se advierten dos problemas. En términos generales, se atiende al vacío historiográfico en esta área de conocimiento: por fuera de publicaciones de corto alcance, no se cuenta con trabajos que recopilen, evalúen y analicen estas experiencias desde una perspectiva político-pedagógica. Para el caso indagado, a lo dicho se acopla la presencia de significaciones histórico-pedagógicas equívocas y/o inferencias conceptuales que contradicen sus principales rasgos, según puede seguirse en las fuentes rastreadas, principalmente aquellas de orden documental. Con lo dicho, se busca visibilizar las condiciones históricas que posibilitaron su emergencia desde finales de 1950; trazar sus coordenadas curriculares generales, y recepción sociocultural a los efectos de profundizar los conocimientos del particular y campo histórico en el cual se inscribe.
\end{abstract}

\section{Palabras clave}

Educación no formal - Universidad Popular - Biblioteca Vigil de Rosario.

* Profesora en Ciencias de la Educación. Universidad Nacional de Rosario. Becaria doctoral del CONICET. Doctoranda en Educación. Facultad de Ciencias de la Educación. Universidad Nacional de Entre Ríos. 


\begin{abstract}
This article aims to describe and discuss the experience of the Popular University ("Library Vigil") established in 1964 and closed in 1977 by the civilian-military dictatorship in Argentina. Completed the investigation about the history of the entire organization, it is considered important to present the main (quantitative and qualitative) characteristics in order to understand historically its non-formal educational projects. In this regard, two problems are noted. Historiographically considered, this area of knowledge presents a significant void: except for short-range publications, there are no reviews and analysis that systematize these educational experiences from a political and pedagogical view. In the case inquired, also warned the presence of mistaken historical-pedagogical significations, and / or conceptual hypotheses that contradict their principal features, as can be followed on the traced sources; mainly written sources. As such, it seeks to make visible the historical conditions that enabled its emergence from late 1950; follow their curricular designs, describe the social and cultural reception to further the knowledge of the case and historical field.
\end{abstract}

\title{
Key words
}

Non-formal Education - Popular University - "Library Vigil”, Rosario.

\section{Génesis institucional}

Los inicios de la Biblioteca Popular "Constancio C. Vigil" se remontan al año 1944 cuando se crea una pequeña biblioteca en la Asociación Vecinal del barrio "Tablada y Villa Manuelita" (Rosario), previamente inaugurada en 1933 al calor del movimiento asociaciacionista en marcha desde la década del '20 y hasta mediados de los ' 40 . Combatidas y replegadas las acciones a-sistémicas y contestarias de principios de siglo (fundamentalmente de las tendencias anarquistas), estas organizaciones barriales fueron expresiones de una sociedad de entreguerras orientada a la negociación para el progreso de las capas bajas de la urbe; mejor decir, adquirieron mayor visibilidad a los efectos de negociar sus intereses fomentistas con la administración del poder. En dicho proceso, se tornaron verdaderos agentes de socialización en su contexto inmediato; no obstante la diversidad de actividades y objetivos particulares, vecinales y bibliotecas en su conjunto respondieron a un significante de época que cobró intensidad y estabilidad: "elevar la cultura" (1). Esta expresión tomó formatos y prácticas materiales precisas: ciclos de conferencias a cargo de especialistas en amplios y diversos temas de actualidad, lecturas colectivas, concursos de poesía, dibujo y pintura; entre otros. La moderación, la prudencia y la buena voluntad fueron por entonces las notas (espiritualistas) dominantes, asimismo desprendidas de las formas "cultas" del entretenimiento que también dejaron su huella en el ámbito privado, forjando un espacio doméstico para los libros "serios" y revistas pasatistas. 
En particular, el barrio "Tablada" se pobló de las típicas entidades populares construidas para la participación popular, la ayuda mutua y la socialización cultural urbana. Vecinales, clubes y bibliotecas, ganaron lugar en el paisaje chato y rutinario del sur rosarino. Para el caso que se sigue, entre los años 1953 y hasta 1959, un entusiasta grupo de adolescentes y jóvenes se sumó al ámbito vecinalista conformando una Sub-comisión de biblioteca (S.C.). Ciertamente, su presencia revigorizó el espacio con prácticas y saberes que continuaban la línea sociocultural del asociacionismo barrial (2), al tiempo que también evidenciaron acciones comunitarias que se ya desviaban de la matriz fomentista (3) y/o articulando su quehacer con la vida escolar de la aledaña escuela "República del Perú".

Ahora bien, en torno de indicadores que prefiguraron la génesis de Biblioteca Vigil, resulta prioritario subrayar el inicio de la exitosa rifa lanzada por el grupo juvenil en 1956, y más aun, su modalidad de financiamiento "en cuotas" desde 1958. Al respecto, los testimonios coinciden al señalar que el joven Augusto Duri (4) ideó este particular (y por entonces inédito) mecanismo, pues, según recuerda el ex tesorero Tomás Pedrido: "Hablar en esa época de una rifa en cuotas...y era una cosa de locos" (5). Efectivamente lo era por su modalidad de pago pero también por su articulación simbólica: el mundo pagano de la apuesta - el negocio del juego - acoplado a la sagrada esfera de la cultura (6).

La rifa fue exitosa desde su implementación; en pocos años su crecimiento fue sostenido y exponencial (7). Pero incluso antes de generar ingresos millonarios, "la-rifa-de-la-Vigil" posibilitó definitivamente la institucionalización de "la Vigil". Ella no sólo resultó un sólido y potente motor financiero sino que sobrevino desde intereses generacionales, políticos y pedagógicos que ya se desanudaban y autonomizaban del ámbito vecinalista. En este sentido, condensa lo intrínsecamente popular de la organización en ciernes: lo impuro cultural (8); la combinatoria de unos horizontes espiritualistas encarnados en inéditos dispositivos materiales que sin pausa se volcaron a los sectores menos favorecidos (9). En suma, encontrados los recursos financieros e identificados los (propios) objetivos organizacionales, en 1959 la Sub-Comisión se separa de la vecinal y nace como asociación civil "Constancio C. Vigil". En adelante, sus proyectos crecieron y desarrollaron de forma embrionaria; esto es, la génesis de cada ámbito fue fruto del cruce de un específico interés grupal y/o particular y las reales posibilidades de efectivizarlo de forma autogestionaria, racional y participativa (10). Desde unas prácticas altruistas del tiempo libre, la humilde biblioteca se fue transformando en una institución de sólido capital financiero y patrimonial que entonces demandó su dedicación completa, finalmente plasmada en una comisión directiva (C.D.) estable cuyos miembros continuaron en igual funciones hasta 1977 por vía del voto mayoritario de la masa societaria. Llegada la década del '70, resultó un complejo social, cultural, educativo y mutual sostenido por 19.639 socios, con 647 empleados y con 2.956 alumnos en sus escuelas primaria, secundaria, jardín de infantes y universidad popular. 


\section{La historia "contada". Universidad y académicos populares}

Aunque las Universidades Populares tienen una vasta trayectoria en la historia educativa no formal en nuestro país, dicha impronta no se exhibe historiográficamente. Por fuera de publicaciones de corto alcance limitados a breves datos de rigor (ubicación, período, autoridades, etc.), no se cuenta con trabajos que recopilen, evalúen y analicen estas experiencias desde una perspectiva político-pedagógica. Desde ya, este vacío no habilita presentar a la U.P. de Biblioteca Vigil como un caso desarticulado de referencias anteriores y/o contemporáneas (11). Asimismo, la misma exhibe una génesis anterior a su oficialización en el año 1964, pues cronológicamente acompaña los primeros años de la organización (1959-1963) cuando en su seno se gestaron cursos de enseñanza informal: folklore, ajedrez, títeres, teatro, cine y charlas de divulgación astronómica. Ciertamente, conviene detenerse en ese período atendiendo a la observación de A. Duri: “...la historia 'historia', no está bien contada". Argumenta entonces que:

(...) se han tomado algunos personajes como... hay un montón de compañeros que han iniciado todo esto y son los que realmente estuvieron de los comienzos, y los comienzos... no estamos hablando ni del '70 ni del ' 60 , sino que estamos hablando de la década del '50.

Se coincide con la mirada del ex dirigente al advertir que algunas publicaciones arrojan datos imprecisos y/o conceptos erróneos o atemporales al caso. Por citar dos ejemplos: por un lado, Alderete (2010) describe "El concepto de enseñanza que circulaba en la institución tenía fuertes connotaciones políticas e ideológicas críticas" (2010, p. 96). Aun cuando esta descripción resulta políticamente nebulosa y epistemológicamente polisémica, se destaca en tanto se trata de una afirmación que se contextualiza explicitando: "Durante el período de Lanusse (1971-73), las experiencias de educación popular, originadas en los '60 revivieron, configurándose una pedagogía sintetizada de las experiencias de la Escuela Nueva, de Paulo Freire y de la izquierda" (2010, p. 74). Por otro lado, puede seguirse el trabajo de Tavella (2007); allí se define a la entidad como "una organización civil liderada por intelectuales en su mayoría de fuera del barrio (...) apoyado por gente de muy diversas procedencias políticas y geográficas" (2007, p. 123). Sobre lo último, es importante subrayar que el corpus analizado en la investigación desde la cual este artículo se desprende, arroja un dato contrario e inequívoco: Biblioteca Vigil y esta Universidad en particular fue liderada por gente del barrio y apoyada (y en menor medida gestionada) por intelectuales de muy diversas procedencias políticas y geográficas. Respecto de las descripciones de Alderete (2010), precisamente, las expresiones de A. Duri buscan desanudar esos significantes políticos de finales de los ' 60 , y más aun de la década del '70 homologados bajo inferencias automáticas. Si se prefiere, y siguiendo la expresión del ex dirigente institucional, "no estamos hablando" 
de pretensiones político-pedagógicas de corte revolucionario ni de proyectos informales de carácter a-sistémico en un sentido ideológico radicalizado, pues (aun) "estamos hablando" de la potente idea del bien común continuada desde el apogeo asociacionista y resignificada en la trama de una organización que adquiere una capacidad de financiamiento sin igual, asimismo enmarcada en un contexto barrial filo-peronista que no debe relegarse en el análisis. Puede decirse que las aclaratoria de A. Duri interpela un relato que ha olvidado esta prehistoria (que no es tal) entronando unos actores y perspectivas políticas que en rigor emergen en una etapa ulterior de la organización, y que incluso tampoco serán dominantes sino que empalmarán con la cultura institucional (Bertranou, 2004) bajo una relativa estabilidad, sin exclusión de tensiones y fricciones ideológicas particulares. Sin más, se alude a los años de construcción de la entidad en general y la U.P. en particular, antes de la llegada de Rubén Naranjo (12), ubicándolo aquí como figura referencial del campo académico; extra-barrial; cercano a la izquierda antiliberal post peronismo, y representante de la vanguardia artística local y nacional (13). En síntesis, "la historia "historia" de "la Vigil" y su U.P., es anterior al aporte de la intelectualidad universitaria y vanguardia artística rosarinas que a posteriori imprimieron sobre ella sus propias inquietudes y aspiraciones para la transformación social durante la efervescente década del '60 y turbulentos' 70 .

En virtud de lo dicho, cabe iluminar la (olvidada) presencia del Prof. Rubén Martiniano Rodríguez, primer Director de los nacientes cursos y escuelas de la U.P. Por aquellos años, Rodríguez regenteaba el nivel primario de la Escuela № 615 "República del Perú", aledaña a la asociación vecinal desde la cual surge Biblioteca Vigil. En dicho rol, el profesor abrió las puertas a la joven S.C.; al decir de A. Duri: "la escuela de enfrente y la biblioteca Vigil, eran la misma cosa". Dicha plasticidad tomaba cuerpo en el gran salón escolar depositario entonces de las actividades matrices de los cursos y Escuelas no formales. Desde "la primera hora" y hasta sus últimos días, Rodríguez apoyó con entusiasmo la tarea en marcha que incluía la participación de muchos de sus alumnos. Ciertamente, ello habría proseguido de no ser por una enfermedad que ya lo aquejaba; en palabras de Tomás Pedrido: "iEra un tipazo!, luego tuvo algunos inconvenientes, era hermano de un cantor muy importante que tenía D'Arienzo, era bárbaro, pero... por ahí le faltaban fuerzas para manejar todo eso". Llegado el año 1963, no estaba prevista la selección de otro director para organizar las actividades en franco crecimiento. Claramente, Rodríguez comulgaba en muchos aspectos con la cultura institucional en ciernes, dato que el ex tesorero construye con las variables: vasta trayectoria de organización escolar, enclave barrial e incluso, simbólicamente, en la referencia a la orquesta de Juan D'Arienzo que interviene en el recuerdo de Pedrido (14). Desde ya, las razones de su renuncia quedan esclarecidas en la expresión "todo eso", refiriendo un trabajo por venir que demandaba no sólo experiencia 
de gestión, sino tiempo y energías significativas. Bajo estas circunstancias, y ya incorporado al staff organizacional, Rubén Naranjo sugerirá el nombre de Teresa Martí, cuya trayectoria abrevaba a las últimas corrientes escolanovistas, pero, todavía más, en tanto la docente acompañaba las características de la obra institucional en su conjunto. Así, la U.P. se configuró durante la etapa de Martiniano Rodríguez y se institucionalizó con la llegada de Rubén Naranjo en 1963 y bajo la dirección de Martí desde 1964.

\section{El mural de Naranjo, las "Pitman" y la Escuela de Artes Visuales}

A mediados del año 1963 el primer edificio construido por la entidad estaba prácticamente terminado. En tanto se culminaban aulas y espacios destinados a la Biblioteca Central y Jardín de Infantes, inmediatamente comenzaba el traslado del material, personal y actividades sociales, culturales y educativas; restaba tan sólo embellecer su fachada. Los arquitectos Liberatore y Aguirre convocaron entonces a Rubén Naranjo. El proyecto estipulado en no más de treinta días consistía en la incorporación de una serie de murales externos e internos. No obstante, el trabajó se extendió por tres meses; algunos materiales fallaron demandando el ensayo de diversas pruebas.

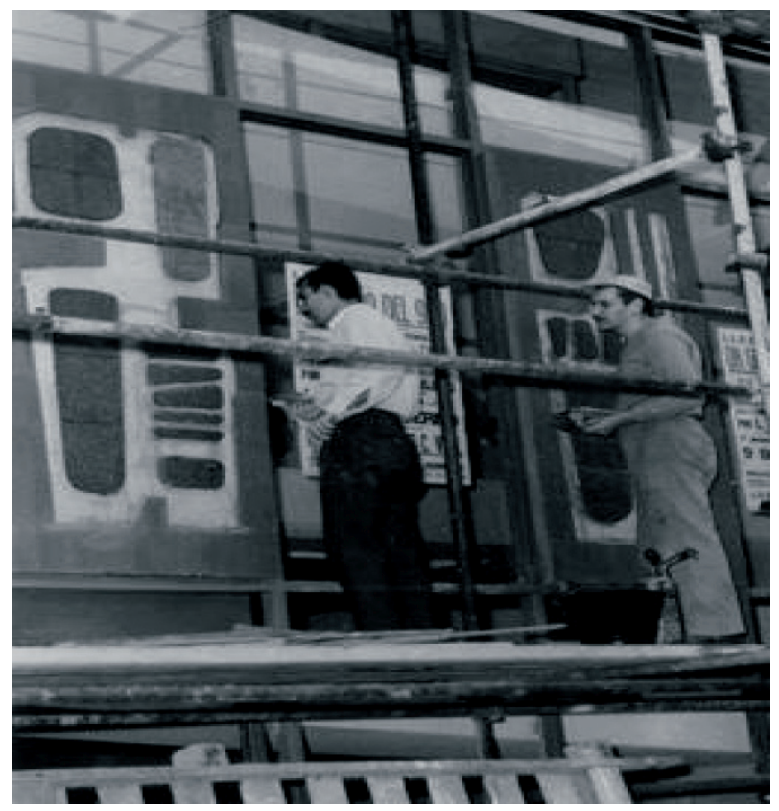

Izq. Osvaldo Boglione. Der. Rubén Naranjo trabajando sobre los murales de la fachada del edificio inaugurado en 1963 (15) 
Como lo deja ver la fotografía, y como bien supo decir el propio Naranjo, los días transcurrieron y la vista "desde los andamios" resultó privilegiada para observar una escena novedosa; siguiendo a Ares (2008), el artista relata un pasaje subjetivo que se sitúa "en ese borde paradójico que separa pero también une y atraviesa vida empírica y obra" (2008, p. 21). Específicamente, se define como "(...) un tipo de izquierda, con un gran respeto por la revolución cubana (...) y antiperonista por experiencia de vida" (citado en Longoni, 2008, p. 362), al tiempo que describe a la entidad expresando: "La Vigil era una institución de barrio, y por lo tanto, netamente peronista; del presidente al telefonista todos eran peronistas, sus mujeres, sus hijos. Yo venía de un espacio netamente antiperonista, tenía otra formación, tenía otra visión" (citado en Longoni, 2008, p. 363). Esta "visión" comienza a ser interpelada cuando Naranjo bajaba de los andamios para: "charlar con ellos (...); tomábamos unos mates" (citado en Oliva, 2004, p. 12). Con entusiasmo y asombro indagaba en las actividades y modos de funcionamiento organizacional: "fui viendo cómo vivía la gente de esta biblioteca, cómo trataban a los chicos, cómo trataban a sus padres" (Ibídem). Sus palabras referirían puntualmente al Jardín de infantes en plena mudanza hacia los nuevos locales bajo la dirección de la Prof. Antonia Frutos. De forma curiosa, ella también se sorprendía del artista a quien confundió con un obrero de entre tantos que levantaban el edificio. Entre risas, en la entrevista la docente comenta que recuerda decir: "ique nivel cultural tiene ese albañil; que bien se expresa!'. En suma, la sensibilidad y el cuidado apreciados pusieron en tensión preconceptos y legados: "(...) descubrí otro peronismo, lo fui viviendo y me fui descubriendo en un mundo distinto a mí mismo. Descubrí que había un espacio del peronismo profundamente compatible con cosas que yo creía" (Ibídem).

Entre tanto se gestaba esa huella silenciosa, Naranjo y Boglione finalmente culminaron la obra. Llegada esa instancia, Augusto Duri lo sorprendió con una invitación: "Quédese. Vamos a hacer una Escuela de Arte. Ésto es lo suyo" (Ibídem). Contrariado, Naranjo respondió: "Pero yo ¿qué puedo hacer aqui? (...) Tal vez sí... se pueda hacer, pero yo me pregunto si es lícito hacerla en el barrio. Si para esta realidad, tiene sentido crear una Escuela de Arte" (Ibídem). En este pensamiento desnudo, la articulación de los conceptos "lícito", "realidad", "sentido" y "arte" dan cuenta de unos supuestos estético-políticos prescriptivos para los sectores populares. En este sentido, si al decir de Frigerio (2007) la educación estética puede operar a modo de fetiche; como una ideología [siguiendo a Eagleton] o ser un anuncio emancipador (2007, p. 26), cabe preguntar ¿Cómo se tramaba aqui? ¿Cómo lo suponía el universitario convocado? El punto es que siquiera aun lo suponía en tanto él mismo, exponente del campo, parecía no tener cabida en el espacio barrial; en rigor, el barrio no tendría cabida en el campo artístico "mayor". Y no acaso por razones ideológico-partidarias en franco proceso de interpelación, sino en rigor de un reparto del saber condicionado por necesidades materiales (supuestamente) 
anteriores. Si allí se ubicaba la inquietud del universitario, precisamente, ¿por qué la vanguardia de aquellos años podía "denunciar" la pobreza pero acaso no intervenir subjetivamente en ella?; mejor interrogar, ¿qué desanuda (en el caso) ese hipotético obstáculo? En este punto, R. Naranjo explicita que A. Duri le brindó una respuesta decisiva; ésta fue: "Mire, a nosotros nos importa crear cosas para que la gente crezca, y si alguien quiere hacer usos prácticos que vaya a las "Pitman" [16] (citado en Oliva, 2004, p. 12). En la entrevista que le realizara Longoni (2008), Rubén modifica levemente las palabras del presidente de "la Vigil": "Nosotros tenemos que hacer lo que haga crecer a la gente, no lo que le haga ganar un mango. En vez de cursos de taquigrafía hagamos cursos de arte" (citado en Longoni, 2008, p. 367). Como fuera, concluye que los dichos de A. Duri "le quedaron grabados" y agrega: "(...) era una reflexión excepcional" (Ibídem).

A todas luces, la comparación entre el proyecto de la U.P. y las afamadas "Academias Pitman" guarda consigo un tono de desprecio. A nuestro entender, el rechazo se orientaba a la mercantilización de las fantasías y necesidades populares y al descrédito de otras inventivas y potencialidades de los vecinos de "Tablada" en particular. Sin embargo, es dable relativizar estas percepciones si se considera la posterior incorporación de cursos de Dactilografía, Mecanografía e Inglés. En una lectura superficial, ello puede interpretarse como una contradicción y/o desvío de sus objetivos espiritualistas; sin embargo, se trata de una negociación que evidencia el "impuro" carácter popular de la institución. Dicho de otro modo, al tiempo que se crean las condiciones que permiten continuar y robustecer la heredada y abrazada oración cívica del asociacionismo ("elevar la cultura de la barrio"), igualmente se atienden y respetan necesidades y aspiraciones materiales y simbólicas que advienen en formatos similares al modelo "Pitman", pero que sin dudas resultan diferenciales en virtud de su ingreso libre y gratuito; mejor decir, en virtud de otras variables curriculares que se expondrán en el siguiente apartado.

Una fuente documental en particular colabora en la perspectiva arriba señalada. Se trata de la encuesta elaborada por los sociólogos Hilda Habichayn y Héctor Bonaparte de la Universidad Nacional de Rosario fechada el 12 de diciembre de 1969. Por entonces, los mencionados profesionales fueron contratados para monitorear la opinión de los asociados en torno de una serie de proyectos institucionales para el año 1970; entre otros: "la ampliación de los Cursos de la Universidad Popular" (1969, p. 9). Para ello, se formularon dos preguntas sencillas; a saber: "¿Qué cursos de la Universidad Popular les interesan? ¿Qué otros cursos harían falta?” (Ibídem). El informe explicita entonces que: "de entre una larga lista de cursos enumerados, los que cuentan con mayor preferencia son" (Ibídem): 


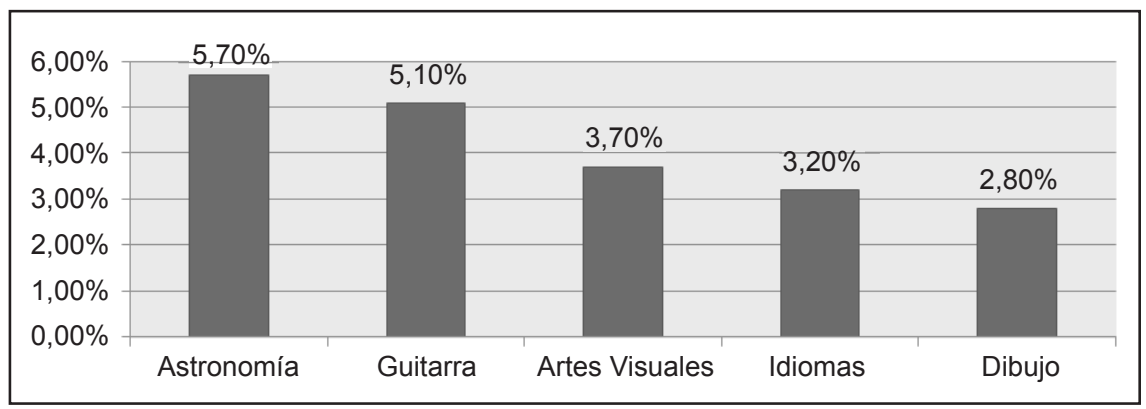

Por su parte: "los cursos solicitados son muchos y diversos. Los más mencionados son" (Ibídem):

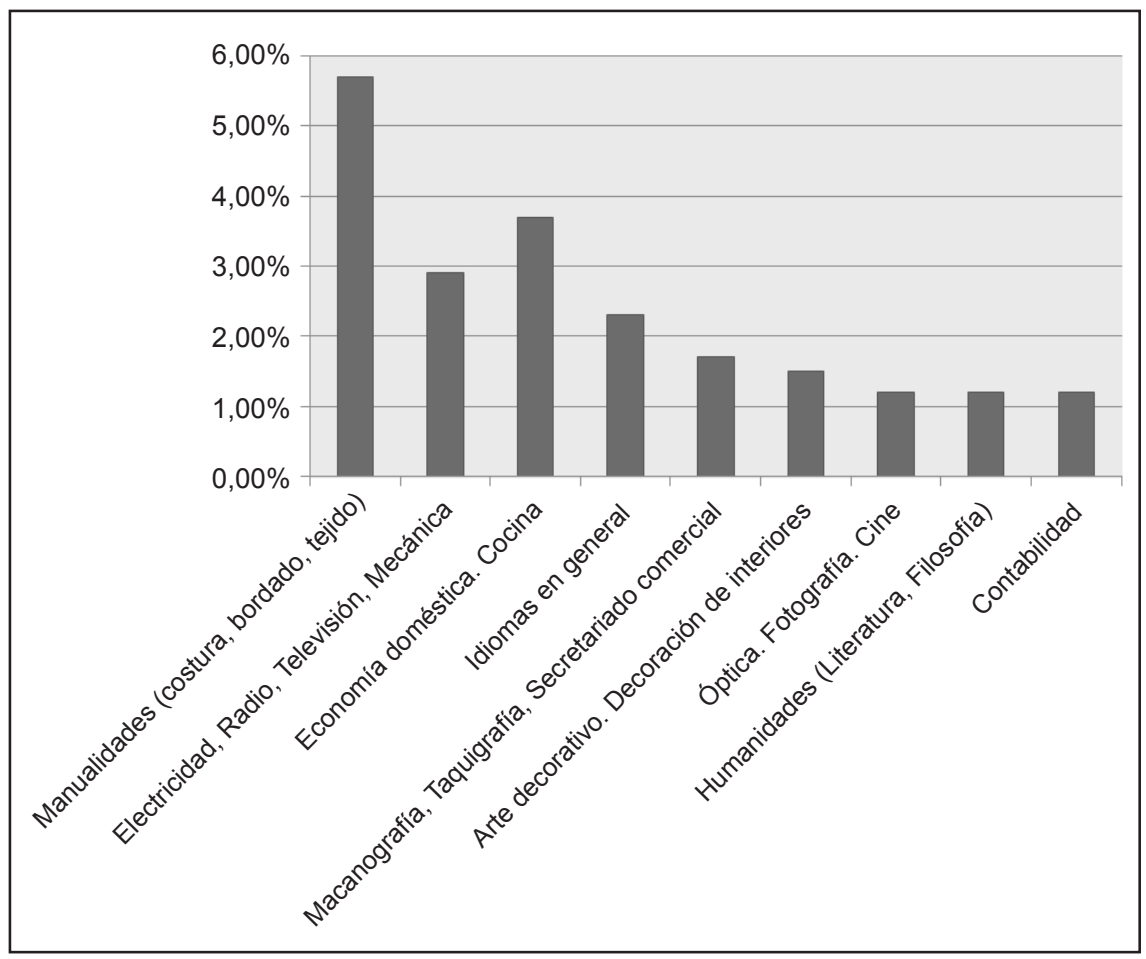

Los pedidos de la masa societaria resultan un universo de prácticas, saberes y oficios largamente ensayados en la historia de la educación popular; algunas guardan variados vestigios conservadores y otros expresan novedades tecnológicas que ya no lo son tanto hacia 1969. Ahora bien, entre las deman- 
das pragmático-populares del espacio barrial y las ansias superadoras de la organización, se cuajaba una batalla cultural que los universitarios observaban en un segundo plano. Desde ya, esta lucha simbólica por el "crecimiento de la gente" resultaría una negociación asimétrica si se apunta que la decisión final respecto de los cursos efectivizados se alojaba en el núcleo dirigencial; en su carácter aduanero. Sin embargo, dicha asimetría no acababa siendo ficcional; vale decir, se registraba una atenta escucha institucional que tomaba nota de un estado de la cuestión; desde allí se intentaba jalonar "hacia arriba" o hacia lo que se considera "arriba" en el desarrollo cultural de los sectores humildes. Pues bien, he allí un poco de Mecanografía, otro tanto de Taquigrafía e Inglés; pero, he allí de forma desproporcionada, la música, la poesía, el arte y el cosmos para abrir y conmover el pequeño mundo de los habitantes del barrio "Tablada". Posiblemente, ésta fuera la "excepcional reflexión" a la que aludiera Rubén Naranjo; quizá comprendiera entonces lo lícito y necesario de esta "cruzada" no limitada ni a la maquinaria fomentista ni la pedagogía lucrativa y expeditiva "para pobres" en plan de integrar, oficio mediante, la ensanchada clase media argentina. Entre una y otra (legítima) aspiración social y cultural, la U.P. de Biblioteca Vigil devino una propuesta orientada a la conmoción de los mezquinos imaginarios introyectados para sí y para el otro.

Por fuera de estas hipótesis, lo cierto es que Naranjo aceptó la apuesta de A. Duri organizando y dirigiendo la flamante "Escuela de Artes Visuales". Desde entonces, sobre ella imprimió su capital cultural: una disciplina del conocimiento, un saber organizacional; una trayectoria, y un conjunto de relaciones personales y laborales. En palabras del artista plástico: "Se incorporaron docentes como Albarello, Rippa, el "negro" Boglione (...) todos íbamos pasando por la escuela de "la Vigil", éramos toda gente de izquierda. Hacíamos nuestro trabajo con gran respeto hacia la gente de la Biblioteca" (citado en Oliva, 2004, p. 7). Asimismo, a raíz de la experiencia "Tucumán Arde" (1968) Naranjo se relacionó con otros exponentes del campo; algunas de esas nuevas amistades también se incorporaron a la Escuela: "(...) se desempeñaban en otras áreas de la biblioteca Raúl Pérez Cantón y Sara López Dubuy. Hacíamos nuestro trabajo con mucha seriedad y ello produjo un efectivo acercamiento con los directivos de la institución". Finalmente, las dudas que pesaban sobre la factibilidad y legitimidad ética y sociocultural del proyecto, se despejaron tempranamente: "Fue una sorpresa para mí. Se inscribió mucha gente (...) gente grande, obreros, laburantes" (citado en Oliva, 2004, p. 10).

\section{Cursos y Escuelas oficializadas}

En adhesión al principio de la educación permanente, la Universidad enriquecida por los académicos "populares" tuvo una mirada o propósito social amplio: "Esta forma de educación permanente no sólo cumple con la premisa de encauzar el tiempo libre, sino que a nivel de la problemática juvenil logra sacar 
a los adolescentes del peligro de la calle, orientándolos hacia una mejor convivencia" (1975, p. 4). Los diseños curriculares efectivizados fueron coherentes al respecto; sus contenidos, criterios metodológicos y evaluaciones, condensaron las ansias de dar una tregua a la alienación del espíritu y al cuerpo agotado tras las rutinarias jornadas en la fábrica, en el puerto, en el cercano frigorífico Swift, en la oficina o tras las mecánicas e inexpresivas tareas domésticas (17). Al respecto, el ex bibliotecario lo apunta con detalles singulares:

(...) no había una promoción por puntaje, por calificación. A la escuela de Bellas Artes ibas tres años, bueno, y hacías primer año todo lo que tenías que hacer, llegabas hasta donde podías llegar, y asi el segundo, y llegabas hasta donde podía llegar cada uno, algunos más, otros menos. Y hacías el tercer año de la misma manera, y se le daba un certificado de que había cursado, simplemente.

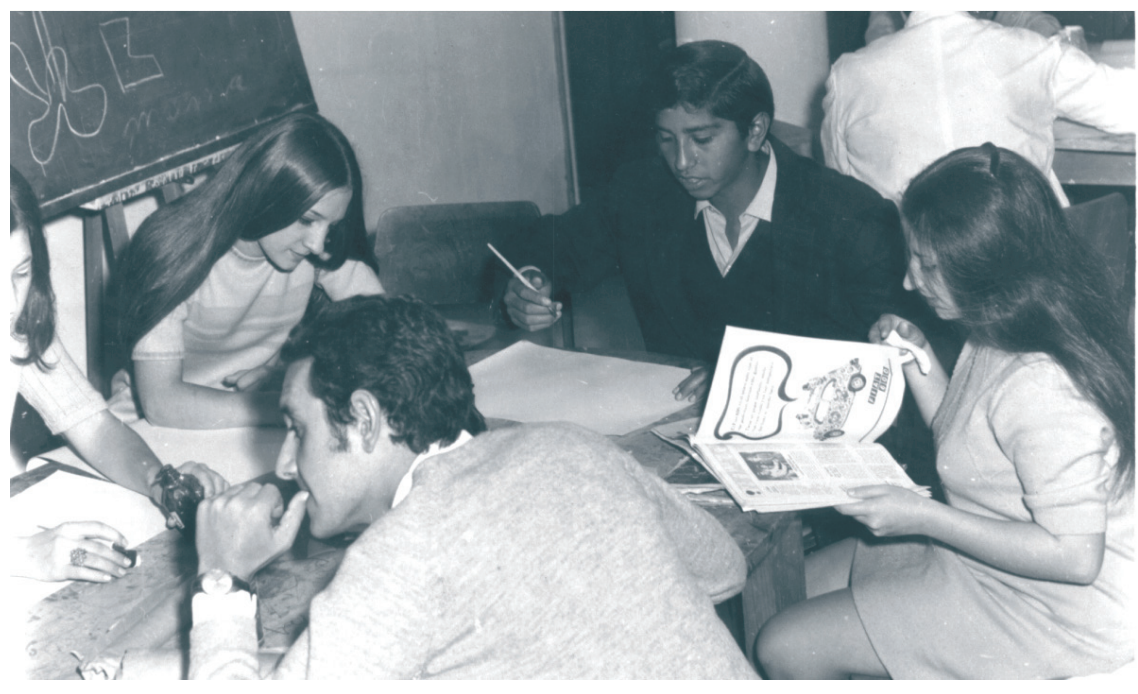

Clase de Dibujo. Escuela de Artes Visuales (Foto: Biblioteca Vigil)

El ex bibliotecario refiere en sus dichos a la Escuela de Artes Visuales dirigida por R. Naranjo e integrada por los cursos de: "Pintura", "Grabado", "Dibujo", "Escultura", "Cerámica" e "Historia del arte". Dependiendo del caso, los cursos tuvieron una duración de tres o cuatro años. Dada la población que asistía y a la cual se orientaba, funcionaron en horario nocturno (desde las 20.00 y hasta las 23.00 horas) todos los días hábiles a excepción de "Cerámica" que también se dictaba los sábados por la tarde y domingos por la mañana. Para esta Escuela en particular se requirió una edad mínima de 16 años y 6to grado 
aprobado. A posteriori, la apertura del taller "Expresión creadora infantil" y "Taller experimental", compensó el desfasaje etario en tanto allí asistieron niños desde los 6 años de edad. Hacia el ciclo lectivo 1975, 145 niños se inscribieron en ellos (sólo para dicho año).

Por otro lado, y como todos los emprendimientos culturales, educativos, sociales y económicos forjados por la entidad, las condiciones edilicias, disponibilidad y calidad de los dispositivos didácticos necesarios, fueron especialmente atendidos y provistos, superando en algunos casos la media que por entonces existía en otras instituciones públicas o privadas con fuertes aranceles de ingreso y permanencia. Entre otros ejemplos, Raúl Frutos describe que "(...) [las escuelas] tenían las mejores condiciones, dábamos Cerámica y por ahí pusimos un horno como no había otro en Rosario. La capacidad, la calidad... se hacía todo el material necesario para trabajar". Asi, la provisión de espacios ventajosos provistos de una alta calidad tecnológica (18), sumados al ingreso gratuito e irrestricto y la flexibilidad del diseño del cursado, promovió un encuentro sociocultural y etario diverso e inclusivo. Niños, adultos, ancianos, profesionales, estudiantes y amas de casa concurrieron diariamente a los cursos para "que hagan lo que le gusta, un lugar para que la gente pueda dedicar sus esfuerzos, sus ganas, sus inquietudes. (...) juntamos a chicos de quince años con jubilados de setenta" (R. Frutos).

Otro efecto auspicioso se vio proyectado en términos vocacionales e incluso ocupacionales, por cuanto muchos vecinos pudieron des-cubrir el goce de un saber o encauzar otros añorados pero inalcanzables materialmente, volviéndose en el tiempo una herramienta de trabajo. Nuevamente el ex bibliotecario apunta:

(...) otro caso típico era la enseñanza de la música; había cursos del más alto nivel por ejemplo de flauta traversa, tal es asi que ahora hay un alumno que estudió flauta traversa en "la Vigil", que hace años que está en Alemania y vive de la música, vive de la flauta traversa $(. .$.$) si se daba guitarra se daba un curso teórico con todas las$ especificaciones de la enseñanza clásica de la música, y se daba también un curso para el que quería "rascar" la guitarra, para acompañarse a cantar. No era un criterio escolástico sino que la gente hiciera lo que le gustara en el mejor ámbito posible, para que pudiera desarrollar sus habilidades, sus conocimientos, sus inquietudes.

Esta Escuela de Música abrió sus puertas en abril de 1963; de forma paulatina se fueron integrando la enseñanza de diversas disciplinas hasta alcanzar una currícula comprendida por: "Piano", "Guitarra", "Guitarra acompañante", "Armónica", "Flauta dulce", "Flauta traversa", "Música de cámara con ensambles musicales", "Armonía" y "Educación musical", que asimismo comprendía: "Fundamentación teórica", "Análisis", "Solfeo" e "Historia de la música". 


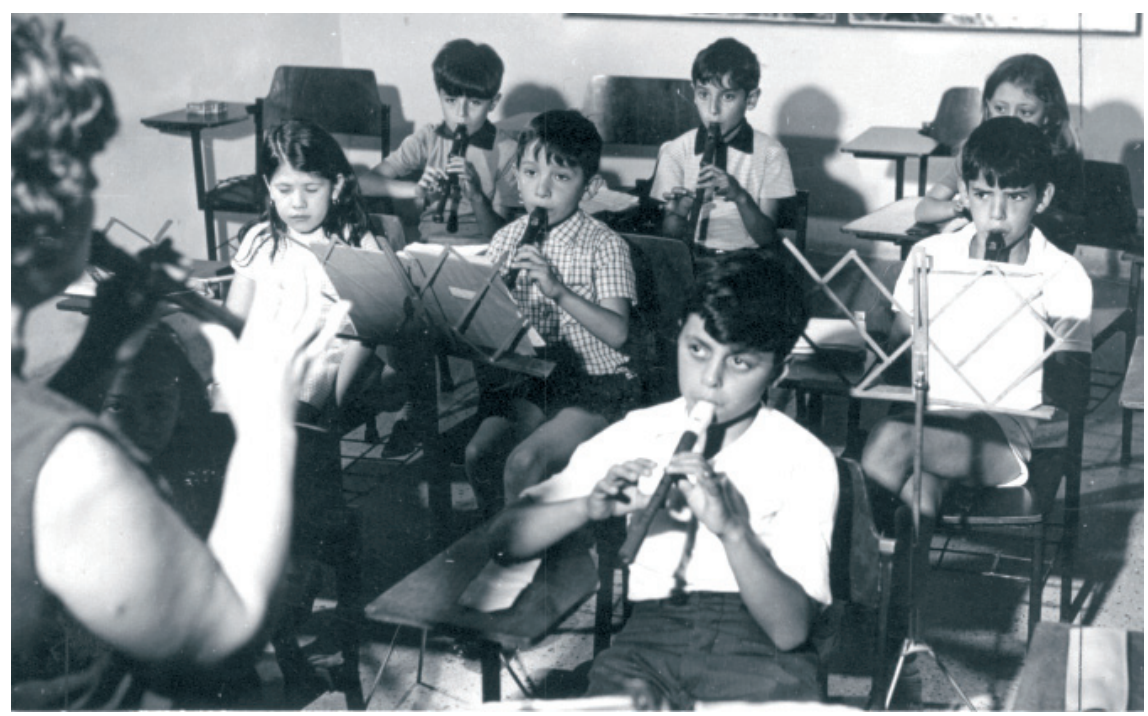

Clase de flauta dulce. Escuela de Música (Foto: Biblioteca Vigil)

Su concreción sobrevino a un ritmo vertiginoso hasta alcanzar una organización y ordenación estable en términos de estructura y variable en contenidos. Específicamente, el primer año comenzó con el dictado de un "Curso Preparatorio" en el cual se inscribieron 42 niños y 16 adultos, y un $1^{\circ}$ año con 10 alumnos entre jóvenes y adultos. Siguiendo las fuentes documentales (Memoria Institucional, 1972), los ciclos lectivos 1964 y 1965 pueden desgranarse y volcarse en el gráfico confeccionado:

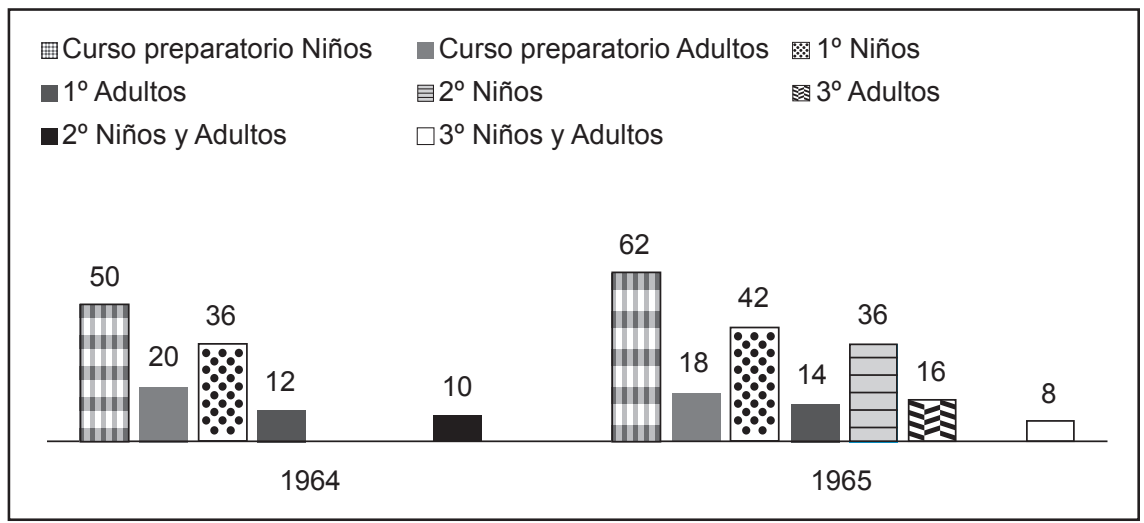


Hacia 1967 "se duplican aproximadamente los alumnos" (1972, p. 7). En 1969, "se agrega la cátedra de piano", y el ciclo lectivo se cierra con: Guitarra (128 alumnos y 3 profesores); Piano (36 alumnos y 1 profesor); Historia de la música y Fundamentación teórica (164 alumnos y 1 profesor). En 1969 se agrega "Flauta traversa", y en 1972 todo lo dicho continúa al tiempo que "se agregan como alumnos en Fundamentación teórica los integrantes del Coro Mixto Rosario Juan Untersander" (1972, p. 8). Hacia 1975, la Escuela de música funcionará con los cursos de: Guitarra (260 alumnos); Piano (120 alumnos); Flauta traversa (16 alumnos) y Armonía ( 6 alumnos).

Ahora bien, en tanto algunas actividades tomaron el formato de Escuelas, otras se organizaron a modo de áreas de conocimiento. Sumado a las mencionadas (Escuela de Artes Visuales y Música), se abrió también la Escuela de Teatro y Escuela de Astronomía; esta última incluía el dictado de: "Matemática", "Física", "Química", "Cosmografía", "Biología", "Astronomía práctica", "Trigonometría esférica", "Electricidad" y "Electrónica". Por su parte, la totalidad de las actividades se completó con los cursos de Idiomas, Mecanografía, Dactilografía, Educación Física, Artesanía, Ajedrez, Expresión literaria, Coros y Folclore.

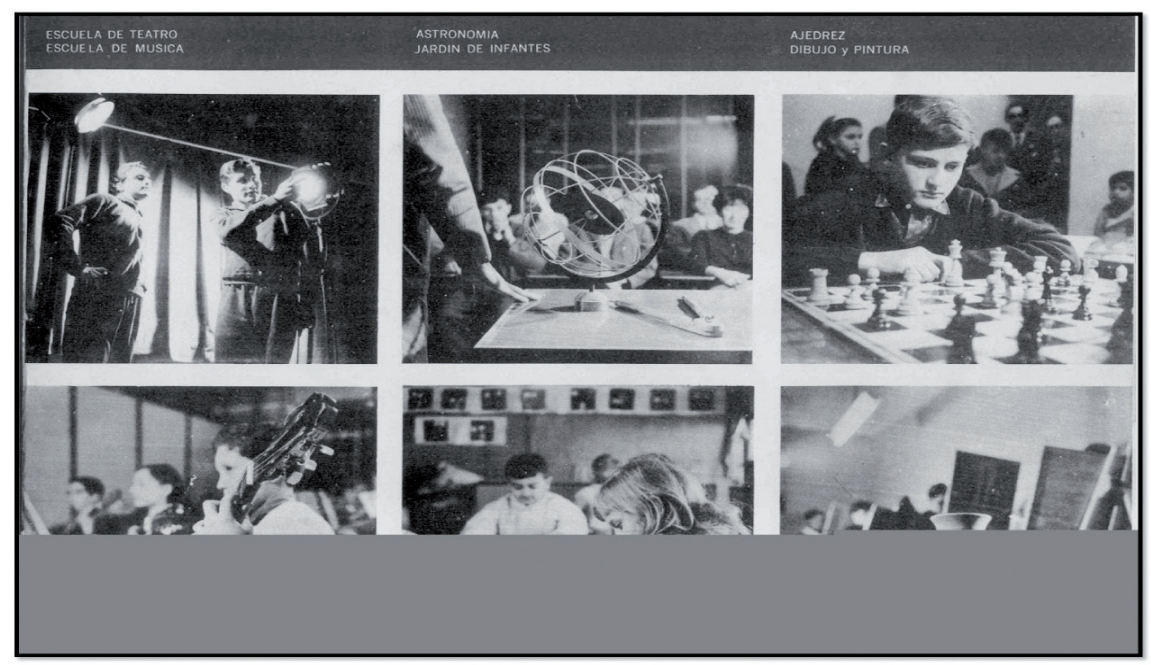

Cursos y Escuelas Universidad Popular (Foto: Biblioteca Vigil)

La única estadística que reúne los datos generales refiere al año 1975. De forma textual (y ello incluye las omisiones observadas), se transcribe el cuadro confeccionado según las fuentes primarias. 


\begin{tabular}{|l|c|c|c|}
\hline \multicolumn{1}{|c|}{ Actividad } & Alumnos & Duración & Categoría \\
\hline Artes Visuales & 44 & 3 & Adultos \\
\hline Cerámica & 46 & 2 & Adultos \\
\hline $\begin{array}{l}\text { Música (Piano; Guitarra; } \\
\text { Armónica; Flauta dulce; } \\
\text { Flauta traversa) }\end{array}$ & 168 & 4 & $\begin{array}{c}\text { Niños y } \\
\text { adultos }\end{array}$ \\
\hline Armonía & 15 & 2 años & Adultos \\
\hline Ed. Musical & 41 & 3 años & Niños \\
\hline Flauta dulce & 23 & 2 años & Adultos \\
\hline Armónica & 17 & 1 año & Adolescentes \\
\hline Guitarra acompañante & 30 & 1 año & Adultos \\
\hline Educación física & 206 & 1 año & $\begin{array}{c}\text { Niños y } \\
\text { adultos }\end{array}$ \\
\hline Artesanía & 70 & 1 año & Adultos \\
\hline Expresión Creadora Infantil & 145 & 1 año & Niños \\
\hline Ajedrez & 20 & 1 año & Niños \\
\hline Inglés & 150 & 5 años & $\begin{array}{l}\text { Niños y } \\
\text { adultos }\end{array}$ \\
\hline Teatro para niños & 117 & 1 año & $\begin{array}{c}\text { Adolescentes } \\
\text { y adultos }\end{array}$ \\
\hline Mecanografía & & & \\
\hline
\end{tabular}

El mismo se completa con el detalle del personal y autoridades a cargo: 1 director (Prof. Teresa Martí), 3 auxiliares de secretaría y 34 profesores especializados. Promediando la década del ' 70 , su estructura y dinámica se volvió estable y aceitada escapando anchamente a los iniciales límites barriales, pues de todos los puntos de la ciudad y sectores sociales llegaban alumnos a la popular Universidad; en 1973 fueron 1.112; en 1974, 1.500; y aun en el turbulento contexto político y crisis financiera nacional y particular de "la Vigil" de 1975, fueron 800 alumnos.

\section{A modo de cierre}

La educación popular gestada por esta U.P. se tramó como una pedagogía orientada al goce estético y la exploración creativa. Los esfuerzos materiales y los objetivos espiritualistas devinieron en un quiebre de los circuitos culturales 
y educativos locales hasta entonces segmentados entre un mundo popular y elitista. Hacia mediados de la década del '60, la novedad de su experiencia llegó urgente a las zonas céntricas de Rosario compuestas por la vanguardia artística "en llamas", por los académicos militantes, denunciantes y/o renunciantes de la Universidad del gobierno autoritario de J.C. Onganía (1966-1970), y el ancho espectro de lo que suele llamarse clase media. Bajo estas circunstancias, "la Vigil" conquistó y apropió un corpus de saber, profesiones, técnicas y posicionamientos político-ideológicos, pero nunca cesó de resignificar, y en ello bien vale decir: controlar, sopesar, decidir y consensuar puertas adentro; esto es: "barrio adentro"; esto es: para el barrio. La muy difundida idea de una entidad ideológicamente polarizada tras la llegada de actores y sectores del campo académico, artístico y/o inscriptos en las miradas escolanovistas de finales de los '60, resulta altamente cuestionada si se sigue las fuentes documentales primarias, e incluso las memorias de sus actores clave. Ciertamente, ninguno de sus proyectos educativos, incluidas las actividades no formales, persiguió un orden sociocultural disruptor; la orientación dominante fue la complementariedad, el suplemento, el aditamento y el apuntalamiento, ofreciendo un corpus de saber y saber-hacer no utilitarista y/o mercantilista, e insistiendo en un tipo de formación humana, integral y de carácter permanente guiado por disciplinas artísticas y científicas mayoritariamente. Todo ello partió del supuesto de una faltante, escasez y/o imposibilidad material del sistema educativo formal y del reparto del capital cultural en general, gestando una pedagogía ligada a la oportunidad de la (re)creación, la creatividad, y la férrea convicción del carácter democratizador de la educación, por vía de unos diseños curriculares coherentes a dichos objetivos. Biblioteca Vigil no intentó ser vanguardia política ni experiencia radicalizada. Fue audaz en términos de su cultura material per se y más aun al disponerla sobre el campo popular. El rol aduanero de su cultural institucional dejó pasar la técnica organizacional y puso reparos a las tendencias rupturistas de las corrientes pedagógicas que deambulaban fuera e intramuros, aspirando a la apertura de nuevos mundos cognitivos sin olvido de los legítimos intereses de los habitantes del barrio "Tablada", por momentos reñidos con los enclaves que suponían el "crecimiento de la gente".

Finalmente, y aun cuando el período que se abre con el terrorismo de Estado en Argentina (1976-1983) no ha sido objeto de análisis en este artículo, resulta insoslayable agregar que en 1977 Biblioteca Vigil fue intervenida por el régimen dictatorial bajo el argumento de regularizar los problemas financieros arrastrados por la entidad desde mediados de 1974. Esta "intervención normalizadora" determinó el cierre de todos los servicios socioculturales, educativos no formales y productivos; entre ellos, la Universidad Popular. A posteriori (abril de 1977), la disposición "normalizadora" se transformó en "liquidadora" dando paso a un desguace patrimonial tal que habilita conceptualizar el proceso en su conjunto como un caso de genocidio cultural (19). Paradójicamente, los 
dichos de un miembro de aquella Comisión Interventora iluminan lo inédito de su experiencia; por aquellos aciagos días, el contador intervencionista Emilio Cancilieri expresó: "El negrito que quiera toca el piano, que se lo compre", en clara referencia a la Escuela de Música que supo albergar a cientos de niños, jóvenes y adultos provenientes de sectores marginados de la ancha urbe. Al decir de Arendt, las palabras del colaborador civil sacan "a relucir la fuerza de todo su significado" (2005, p. 21). Biblioteca Vigil no otorgaba un expeditivo certificado de "Técnico reparador de pianos", sino que invitaba al goce de su aprendizaje sin reparar en un "destino" de clase.

\section{Notas Biblográficas}

(1) Se siguen aquí los trabajos de Gutiérrez y Romero $(1989,2007)$ quienes recortan el análisis en bibliotecas de barrios porteños. Por fuera de una indagación por casos, la férrea convocatoria de este penetrante "espíritu" epocal, asimismo se constata en los estudios de Gravano (1989), Armus (1990), Acha (2004) y Pritivitellio y Romero (2006). Para el ámbito rosarino, la intención estatutaria de "elevar la cultura del barrio" se corrobora en las publicaciones de Nicoletti (1998) sobre la "Biblioteca Popular Homero" de Barrio Refinería (1936-1939), y Rabinovich (2009) para la "Biblioteca Mitre" (1938). Asimismo se expresan casos en otro puntos del territorio nacional, tal y como: "Unión Vecinal de Almada" en la ciudad de Río Cuarto (Córdoba) estudiada por Basconzuelo (2008) y la experiencia analizada por Quiroga (2003) en la "Biblioteca Popular Juventud Moderna" de la ciudad de Mar del Plata entre fines de los años treinta y principios de los cuarenta.

(2) Siguiendo el trabajo de Malla (2006) basado en las actas vecinalistas de la década del ' 40 y ' 50 , pueden citarse los regulares concursos de dibujo y pintura infantiles; funciones de cine y charlas de divulgación.

(3) En la línea de los desvíos, una mirada atenta deja entrever especiales metamorfosis. En primer lugar, la puesta en marcha del "Teatrito de Marta y Jorge" de carácter itinerante. Según recuerda Raúl Frutos [Miembro de la S.C. hasta 1959. Bibliotecario mayor de Biblioteca Vigil y parte de la Comisión Directiva (1959-1977)] "se llevaban espectáculos a las escuelas, se llevaban los teatros de títeres, folclore, etc.". Su hermana Antonia [Miembro de la S.C. hasta 1959. Directora del Jardín de infantes; Secretaria del Departamento de Educación de Biblioteca Vigil y parte de la Comisión Directiva (1959-1977)] agrega "íbamos por los barrios, cortábamos calles y armábamos, instalábamos el escenario en el medio de la calle y entarimados a los costados". En segundo lugar, cabe destacar el trabajo en "Villa Manuelita" apuntalado desde la inquietud del desamparo de la niñez más penetrante allí que en el seno del barrio "Tablada". Antonia expresa "[Albino] Serpi [Precursor de la S.C. hasta 1959. Miembro de Comisión Directiva de Biblioteca Vigil 1959-1977] era siempre el que decía: no sirve que hagamos sólo acá y en la biblioteca, tenemos que abrir en "Villa Manuelita" (...) que trabajáramos de [calle] Alem para allá; allá había más problemas". Expresiones tales como "se llevaba", "íbamos", "instalábamos" o "armábamos", no deben pasar inadvertidas, pues las bibliotecas populares se pensaron como espacios convocantes y efectivos a condición de que, entre sus muros, se concentrara un saber y un "ambiente" que ponderara un halo de compostura. Con ello, el corrimiento físico es también simbólico: el dispositivo epocal se estira, desestructura y rearma en otros escenarios escapando parcialmente al orden endogámico que las caracterizaba. 
(4) Miembro de la S.C. de biblioteca hasta 1959. Presidente de la Comisión Directiva (C.D.) Biblioteca Vigil (1959- 1977).

(5) Miembro de la S.C. de biblioteca hasta 1959. Tesorero de la C.D. de Biblioteca Vigil (1959-1977).

(6) Según el historiador Malla, la S.C. "invitó a la "Biblioteca Mitre" a participar de una rifa a medias, pero éstos no adhirieron por una cuestión conceptual, ya que consideraban que no debían lanzarse a una empresa con fines de lucro" (2006, p. 149). El destacado es nuestro.

(7) En parte, ello se debió a la combinatoria de abonarla en forma accesible, ofrecer atrayentes premios (departamentos, autos $0 \mathrm{KM}$, viajes, etc.) y plasmar los recursos en actividades y servicios sociales, educativos y culturales significativos en su comunidad. Desde otra perspectiva analítica, es dable apuntar que el sistema financiero-institucional se afianzó y aumentó hacia 1965 por vía de la eximición de impuestos otorgado por la provincia de Santa Fe (Decreto 5.912) y por tratarse de una organización sin fines de lucro. A ello se sumará la distribución en diferentes provincias del territorio nacional (San Juan, Mendoza, Córdoba, Entre Ríos, Salta, Buenos Aires, Tucumán y Jujuy). Lo dicho produjo la emergencia de los llamados "Bonos bianuales" (se extendía su duración de 12 a 24 meses o cuotas) posibilitando la previsión presupuestaria de proyectos de envergadura para el año en curso y el subsiguiente. El crecimiento de las ventas resultó palpable en el $11^{\circ}$ Bono del año 1966: la cifra trepó de los 35.000 a los 90.000 Bonos vendidos traducidos en montos millonarios mensuales que ingresaban a "la Vigil" y demandando más de 3.000 vendedores y 500 cobradores en todo el país.

(8) "Las manifestaciones de lo popular que habitualmente puede estudiar un historiador nunca son populares en términos puros (...) porque esa mezcla es lo propio de todo el proceso social y cultural: el conflicto, la coexistencia, la impureza" (Gutiérrez y Romero, 2007, p. 35).

(9) 1963: Inauguración del primer edificio de la entidad para uso del Servicio Bibliotecario, Jardín de infantes y cursos de capacitación popular. 1964: Institucionalización del Museo de Ciencias Naturales (luego Departamento de Ciencias Naturales). 1965: creación del Centro de Cómputos. 1966: Inicio de la Editorial "Biblioteca". 1967: Creación de la Caja de Ayuda Mutua. Proliferación de filiales en el territorio nacional. 1965-1969: Construcción del inmueble principal (7 pisos y dos subsuelos). 1969: Apertura del Observatorio astronómico. 1970: Construcción y apertura del Centro Recreativo y Deportivo "La Colonia". 1970: Inauguración del Departamento de Educación. Inicio del primer ciclo lectivo del Instituto Secundario. 1972: Apertura de la Escuela Primaria.

(10) Por ejemplo: una mera charla de divulgación astronómica a cargo de un vecino aficionado, se complejizó hasta transformarse en el emblemático Observatorio Astronómico, o, los conocimientos de un socio en el campo de la "Taxidermia" devino en el Museo de ciencias naturales más importante de la región. Igual pathos se constata para el caso de la Escuela de teatro: en 1960 se conformó el primer elenco teatral de Biblioteca Vigil a instancias de un vecino apasionado por el teatro llamado Enrique Salvador. Aquello continuó con entusiasmo y hacia 1963 se desarrolló un curso de "Introducción al teatro". Al año siguiente se organizó la Escuela con disciplinas específicas de tres años de duración. Finalmente ésta se incorporó al numen de escuelas y cursos de la U.P.

(11) Para el caso argentino, la primera Universidad Popular se registra bajo el nombre de "Sociedad Luz" y su emblemática "Universidad Popular de la Boca" en 1917 constituida como una Asociación sin fines de lucro. Muy cercano en el tiempo, el movimiento universitario reformista (1918) tendrá sus efectos en la promoción de la extensión académica disparada desde la convicción de articular la vida de los claustros y los sectores medios 
y populares. En rigor de los casos seguidos, resulta evidente que las experiencias observadas en las primeras décadas del siglo XX (Argentina), se anudan a la misma matriz sociocultural y política que produce el surgimiento y proliferación de las bibliotecas populares. En este sentido, puede que no toda biblioteca se diversificara en actividades que devinieran en una Universidad popular, pero indudablemente toda Universidad poseía o compartía por entonces una biblioteca en el marco del movimiento fomentista. En este cruce podemos ubicar a la "Universidad Nacional de Belgrano "Alfredo Fazio" (Buenos Aires) caracterizada por una vasta biblioteca para incentivar la lectura de niños y jóvenes. De forma más evidente, encontramos el caso de la "Universidad Popular de San Martin" (La Plata) fundada en 1940 por el "Centro de Fomento" creado a instancias de su elite barrial. También la llamada "Universidad Popular de Paraná" en 1942 es emergente de una Asamblea de vecinos. Por su parte, otras se propusieron brindar una capacitación en artes y oficios tal y como la "Universidad Popular de Resistencia" (Chaco) fundada en 1929; o acaso surgieron ligadas a figuras del campo intelectual y/o artístico como la "Universidad Popular Alejandro Korn" (Buenos Aires) inaugurada en 1936; año del fallecimiento del filósofo (1860-1936) con el objeto de difundir su legado y pensamiento. En la ciudad de Rosario puede mencionarse la particular experiencia de la "Unión del Profesorado de la Universidad Popular" en el edificio de la Escuela de Enseñanza Normal № 2. Igualmente, bajo la órbita de la Universidad Nacional del Litoral (Santa Fe), en 1938 se agregaron Cursos del "Instituto Social" estructurados como formación profesional.

(12) Profesor de pintura egresado (1958) de la Universidad Nacional del Litoral. Docente en la Escuela de Artes Visuales de Santa Fe y Paraná, regente de la Escuela Provincial de Artes Visuales de Rosario. Prof. de la Facultad de Arquitectura de la Universidad Nacional de Rosario.

(13) En la entrevista realizada por Longoni (2008) el mencionado artista señala que "(...) en la ciudad de Rosario confluían tres grupos donde cada taller (...) tenía una propuesta estética bastante distinta: las chicas (Carnevale, Escandell, Maissonave) hacían estructuras primarias; Renzi, Bortolotti, Gatti y Favario estaban en un planteo más conceptual; Ana María Giménez, Martha Greiner, Miranda Pacheco y Tottis con una propuesta vinculada al pop" (2008, p. 361). Al respecto, R. Naranjo se referencia por fuera de estos agrupamientos. Más allá de nombres y tendencias, éstas y otras propuestas estéticas se congregaron en torno de la potente experiencia política "Tucumán Arde" (1968) de la cual Naranjo formó parte. Sobre esta última puede seguirse el libro aquí referenciado (Longoni, 2008).

(14) T. Pedrido se refiere al conocido cantante Alberto Echagüe.

(15) Fuente: http://www.rubennaranjo.com.ar/vigil.html

(16) Instituciones como las Academias "Pitman" eran símbolo de un tipo de educación popular; o mejor decir, condensaban un disponible de educación no formal de gran prédica entre los sectores populares. Su enorme éxito en ésta y otras tantas barriadas estaba arraigado a unas representaciones sobre la movilidad social: invertir en sus expeditivos cursos de Mecanografía, Dactilografía y/o Redacción comercial, suponía la apropiación de herramientas "útiles" para el campo laboral, fundamentalmente, en torno de la figura del empleado público que formaba parte de estos sectores.

(17) "Los adultos, que por centenares pueblan las aulas en el turno de la noche llegan buscando una salida agradable para la necesidad espiritual de abrir el camino a una vida interior más fructífera". En Informe "Departamento de Educación" (1975, p. 5).

(18) En particular ello se condensa en el caso de la lente del Observatorio Astronómico montado en la cúpula del principal inmueble de la entidad, y nacido de la Escuela de 
Astronomía de la U.P. Hacia finales de la década del '60, la entidad adquirió el instrumental en la afamada óptica "Zeiss" de Alemania Federal; ciertamente, inédito en su tipo y campo disciplinar: un telescopio refractor de $150 \mathrm{~mm}$ de diámetro y de $1850 \mathrm{~mm}$ de distancia focal, con reflector espejo parabólico de $300 \mathrm{~mm}$ de diámetro y $3200 \mathrm{~mm}$ de distancia focal, que permitía obtener un aumento en 600 veces.

(19) Esta problemática puede seguirse en García (2012).

\section{Referencias Bibliógraficas}

- Acha, O. (2004). Sociedad civil y sociedad política durante el primer peronismo. Desarrollo Económico, 44 (174), 199-230.

- $\quad$ Alderete A. (2010). Las aulas, los patios: fueron construidos para dignificar al hombre, la dictadura militar les cambió el destino... Revista de la Escuela de Ciencias de la Educación, 5, (4), 95-112.

- $\quad$ Arendt, H. (2005). De la historia a la acción. Buenos Aires: Paidós.

- $\quad$ Ares, M. C. (2008). La memoria y el exilio en El común olvido de Sylvia Molly. En A. M. Zubieta (Comp.). Tramas literarias y políticas: el pasado en cuestión. Buenos Aires: Eudeba.

- $\quad$ Armus, D. (1990) (Comp.). Mundo urbano y cultura popular. Buenos Aires: Sudamericana.

- Bertranou, J. (2004). Notas sobre el concepto de memoria institucional. En J. Bertranou, J. M. Palacio y G. M. Serrano. El país del no me acuerdo (des)memoria institucional e historia de la política. Buenos Aires: Prometeo.

- $\quad$ Frigerio, G. (2007). Grülp. En G. Frigerio y G. Diker (Comps.). Educar (sobre) impresiones estéticas. Buenos Aires: Del estante editorial.

- García, N. (2011), La intervención cívico militar sobre la Biblioteca Popular "Constancio C. Vigil" de Rosario (1977-1980). Un caso para analizar las continuidades y desvíos de la política educativa procesista. Anuario de la Sociedad Argentina de Historia de la Educación, (11), 135-158.

(2012) Una agenda pendiente: delitos culturales y económicos durante la última dictadura militar argentina. El caso "Biblioteca Vigil" de Rosario, Argentina (19772011). Revista Información, cultura y sociedad, (26) (En prensa).

- Gravano, A. (1989). La cultura de los barrios. Buenos Aires: CEAL

- $\quad$ Gutiérrez, L. y Romero, L. A. (1989). Sociedades barriales, bibliotecas populares y cultura de los sectores populares: Buenos Aires 1920-1945. Desarrollo Económico, 29, (113).

Siglo XXI Editores.

(2007). Sectores populares, cultura y política. Buenos Aires:

- $\quad$ Longoni, A. (2008) Vanguardia y modernización. Tucumán Arde. Entrevista a Rubén Naranjo. En A. Longoni y M. Mestman. Del di Tella a "Tucumán Arde. Vanguardia artística y política en el '68 argentino. Buenos Aires: Eudeba.

- Malla, J. (2006). El barrio Tablada y los orígenes de la Biblioteca Vigil. Rosario: Asociación Vecinal Rosario Sud Este.

- $\quad$ Nicoletti, Ma. J. (1998). Entre el baile popular y el match de ajedrez. Los espacios de sociabilidad urbana de los años treinta. El caso de la Biblioteca Popular "Homero" de Barrio Refinería. Rosario 1936-1939. Seminario Regional, Escuela de Historia, Facultad de Humanidades y Artes, U.N.R., Mimeo, Rosario.

- Oliva, A. (2007). Entrevista a Rubén Naranjo [2004) incorporada en El fusilamiento de Penina, reedición en Barcelona: Editorial El viejo topo. 
- $\quad$ Pasolini, R. (1997). Entre la evasión y el humanismo. Lecturas, lectores y cultura de los sectores populares: La Biblioteca Juan B. Justo de Tandil, 1925-1945. Anuario IEHS, (12), 373-401.

- $\quad$ Pritivitellio, L. y Romero, L. A. (2006). Organizaciones de la sociedad civil, tradiciones cívicas y cultura política democrática: el caso de Buenos Aires, 1912-1976. Revista de Historia, (1), (1).

- Quiroga, N. (2003). Lectura y política. Los lectores de la Biblioteca Popular Juventud Moderna de Mar del Plata (fines de los años treinta y principio de los cuarenta). Anuario IEHS, (18), 449-474.

- Rabinovich, S. (2009). Una avanzada en el barrio: José María Torrejón y el inicio de los Ciclos Artísticos, Científicos y Culturales en la Biblioteca Mitre. Avances del Cesor, (VI), 6, 65-79.

- Tavella, A. M. (2007). La Vigil: un caso de identidad institucional regional. En A. M. Tavella. Identidad colectiva: el caso Rosario desde las perspectivas sociológicas y filosóficas. Rosario: UNR Editora. 